\title{
Alzheimer's Disease Neuroimaging Initiative Auditory Verbal Learning Test Functional Test
}

National Cancer Institute

\section{Source}

National Cancer Institute. Alzheimer's Disease Neuroimaging Initiative Auditory Verbal

Learning Test Functional Test. NCI Thesaurus. Code C102112.

The Alzheimer's Disease Neuroimaging Initiative's (ADNI) version of the Auditory Verbal Learning Test (AVLT Version A). This version is modified from A. Rey in 1964 and is used to evaluate verbal learning and memory abilities in both children and adults. The test format consists of a list of 15 words that the patient recalls during 5 separate trials. A second list with 15 new words is read and the patient must recall the words in one trial. Immediately following this task, the patient must recall the words from the first list; then recall them again 30 minutes later. The total number of words and intrusions is tallied for each trial. 